\title{
Translations and Abbreviations
}

Where a reliable English translation exists, I have used it, in some cases with supplementary references to original texts. The translations of Schleiermacher's letters and the Confidential Letters on Lucinde are mine; those of the Soliloquies are from the manuscript of Terrence Tice's forthcoming translation of that work to be published by Edwin Mellen Press. When Schleiermacher's letters appear in both the old and new editions of his correspondence, to the extent that the latter is available, I have cited both sources.

\section{PART I. SCHLEIERMACHER}

BO Brief Outline of the Study of Theology. Trans. Terrence Tice. Richmond: John Knox Press, 1966.

BR Aus Schleiermachers Leben in Briefen. 4 vols. Ed. Ludwig Jonas and Wilhelm Dilthey. Berlin, I858-63.

BW Briefwechsel 1775-1796. 2 vols. Ed. Andreas Arndt and Wolfgang Virmond. KGA V.I, V.2. I 985 , 1988.

BZ Schriften aus der Berliner Zeit 1796-1799. Ed. Gunter Meckenstock. KGA I.2. 1984 .

$\mathrm{H}$ Hermeneutik: Nach den Handschriften. Ed. Hans Kimmerle. Heidelberg: Carl Winter, Universität-verlag, I974.

HM Hermeneutics: The Handwritten Manuscripts. Ed. Heinz Kimmerle. Trans. James Duke and Jack Forstman. Missoula, Mont.: Scholars Press for The American Academy of Religion, I977.

KD Kurze Darstellung des theologischen Studiums zum Behuf einleitender Vorlesungen entworfen. SW I. I. I 843 . 
KGA Kritische Gesamtausgabe. 7 vols. to date. Berlin, New York: de Gruyter, I980-.

L Schlegel, Friedrich. Lucinde in Dichtungen. Ed. Hans Eichner. Munich: Schöningh, 1962.

LF - Friedrich Schlegel's Lucinde and the Fragments. Trans. Peter Firchow. Minneapolis: University of Minnesota Press, I97I.

M Monologen: Eine Neujahrsgabe [Soliloquies]. Kritische Ausgabe. Ed. Friedrich M. Schiele. Leipzig: A. von Hermann Mulert, I9I4.

OR On Religion: Addresses in Response to Its Cultured Critics. Trans. Terrence Tice. Richmond: John Knox Press, 1969.

SW Schleiermachers Sämmtliche Werke. 3 I vols. Berlin: G. Reimer, I 834-64.

UR Über die Religion: Reden an die Gebildeten unter ihren Verächtern. Ed. Georg C. B. Punyer. Braunschweig: E. U. Schwetschke, I 879.

VB Vertraute Briefe über Friedrich Schlegels Lucinde [Confidential Letters on . . . Lucinde]. SW III. I. I 846 .

\section{PART II. COLERIDGE}

BL Biographia Literaria. I vol., 2 parts. Ed. James Engell and Walter Jackson Bate. CC VII. I983.

CC The Collected Works of Samuel Taylor Coleridge. Bollingen Series 75. I6 vols. London: Routledge \& Kegan Paul; Princeton: Princeton University Press, 1969-.

CIS Confessions of an Inquiring Spirit. Ed. H. StJ. Hart. Stanford, Calif.: Stanford University Press, 1956.

F The Friend. 2 vols. Ed. Barbara E. Rooke. CC IV. I969.

LPR Lectures 1795: On Politics and Religion. Ed. Lewis Patton and Peter Mann. CC I. I97I.

LS Lay Sermons. Ed. R. J. White. CC VI. I972.

$\mathrm{N} \quad$ The Notebooks of Samuel Taylor Coleridge. 3 vols. Ed. Kathleen Coburn. Vols. I and 2, New York: Pantheon, 1957, 196I; Vol. 3, Princeton: Princeton University Press, I973.

PW Poetical Works. Ed. Ernest Hartley Coleridge. New York: Oxford University Press, I9I2.

W The Watchman. Ed. Lewis Patton. CC II. I970.

\section{PART III. FULLER}

CHD (Healey) Dall, Caroline Wells. Margaret and Her Friends. Boston: Roberts Brothers, I 895 .

EAL Essays on American Life and Letters. Ed. Joel Myerson. Schenectady, N.Y.: College \& University Press, 1978.

LMF The Letters of Margaret Fuller. 5 vols. Ed. Robert N. Hudspeth. Ithaca: Cornell University Press, 1983-.

MMF Memoirs of Margaret Fuller Ossoli. 2 vols. Ed. R. W. Emerson, W. H. Channing, and J. F. Clarke. Boston: Phillips, Sampson, and Co., I 852.

NYT New York Tribune i 846-50 [microfilm].

SEC "A Short Essay on Critics." EAL $5 \mathrm{I}-57$.

WNC Woman in the Nineteenth Century. EAL 82-239. 


\section{Delicate Subjects}


